\title{
A closed-form and explicit analytical model for crosstalk in CMOS photodiodes
}

Beatriz Blanco-Filgueira, Paula López Martínez, and Juan Bautista Roldán Aranda

Version: accepted article

\section{How to cite:}

Beatriz Blanco-Filgueira, Paula López Martínez, and Juan Bautista Roldán Aranda (2013) A closedform and explicit analytical model for crosstalk in CMOS photodiodes. IEEE TRANSACTIONS ON ELECTRON DEVICES, 60(10), 3459-3464.

Doi: $10.1109 /$ TED.2013.2276748

\section{Copyright information:}

(C) 2013 IEEE. Personal use of this material is permitted. Permission from IEEE must be obtained for all other uses, in any current or future media, including reprinting/republishing this material for advertising or promotional purposes, creating new collective works, for resale or redistribution to servers or lists, or reuse of any copyrighted component of this work in other works. 


\title{
A closed-form and explicit analytical model for crosstalk in CMOS photodiodes
}

\author{
Beatriz Blanco-Filgueira, Paula López Martínez, and Juan Bautista Roldán Aranda
}

\begin{abstract}
A closed-form and explicit 2D analytical model for crosstalk (CTK) effects in p-n ${ }^{+}$CMOS photodiodes for pixel design optimization has been developed in this work. This model complements and extends a previous development describing the photocurrent due to the active area illumination along with the lateral depletion region and lateral components owing to the diffused photocarriers from the surroundings of the junction. The model has very few fitting parameters since it is physicallybased. In this way, it can be of great use for CMOS image sensors designers, especially to fulfill high resolution and small area requirements by pixel size reduction. The model was validated extensively through device simulations with ATLAS and experimental data, and describes the CTK dependencies on light conditions and physical, geometrical and process parameters.
\end{abstract}

Index Terms-Photodiodes, crosstalk, modeling, simulation

\section{INTRODUCTION}

C ROSSTALK (CTK) is defined as the unwanted output signal of a photodiode (PD) due to photocarriers generated by illumination of another device in its neighborhood. Basically, two components of CTK are commonly defined: the optical and the electrical CTK. The former originates from the photons that penetrate the surface of a PD but reach another device due to a particular incident angle or the use of microlenses. The latter accounts for the diffused photocarriers from neighboring PDs and it is the phenomenon tackled in this work because, unlike the optical component, it is always present in standard fabrication processes.

Methods of reducing both optical and electrical CTK usually involve fabrication process modifications, missing the advantage of using standard processes for CMOS imagers, [?]. Apart from the illumination conditions and the fabrication process, CMOS image sensors CTK also depends on the location of the pixels in the array and on the pixel layout, that is, the arrangement of the electronics and the PD within the pixel area. Some papers have also reported that CTK not only depends on the pixel geometry but also on its size, [?], [?], [?]. Thus, as pixels shrink in order to improve the resolution of the sensor, CTK increases [?]. CTK characterization is not possible by traditional means and basically there are two

This work has been partially supported by the Spanish Government under projects TEC2009-12686 and TEC2012-38921-C02-02 (co-funded by the European Region Development Fund, ERDF/FEDER), by the Xunta de Galicia under project 10PXIB206037PR, by the Junta de Andalucía under project P08-TIC-3580 and by AE CITIUS under the project CN2012/151 of the Xunta de Galicia (ERDF/FEDER).

B. Blanco-Filgueira and P. López are with the Centro de Investigación en Tecnologías de la Información (CITIUS), University of Santiago de Compostela, Santiago de Compostela 15782, Spain.

J. B. Roldán is with the Department of Electronics and Computer Technology, University of Granada, Granada 18071, Spain. different strategies available: one of them, based on the use of masks, blocks the penetration of light in some pixels to measure the response due to the illumination of unmasked pixels, [?]; and the other, based on the so-called spot-scanning technique, illuminates an individual pixel in order to measure its effect in the vicinity, [?]. Although these techniques are not demanding for large PDs, the masking may suffer from diffraction and the spot-scanning requires the use of a small laser spot for the characterization of small pixels. Therefore, alternative strategies, such as the use of device simulators, are essential for the study of CTK without suffering the aforementioned practical limitations, [?], [?].

CTK estimation for pixel design optimization, avoiding fabrication process modifications and costly characterization of test structures, is desirable. However, finding a general analytical expression is challenging because multiple configurations are possible even for the same fabrication process and pixel size, showing different performance in terms of CTK. What is viable, however, is the prediction of CTK in the worst case scenario, that is, when two PDs are close together without in-pixel electronics between them, [?]. Additionally, as light propagation and diffusion phenomena occur in perpendicular directions, finding an analytical solution involves solving a two-dimensional problem. In this respect, there are very few CTK models available and there exists a great need of them from the device designer viewpoint. In this sense, the most remarkable approach is found in [?], where a semi-analytical approximation in terms of the pixel geometrical shape is proposed. Although an analytical solution has been proposed recently, it assumes that there is no diffusion in the lateral depletion region due to modifications in a standard fabrication process, neglecting CTK to a large extent, [?].

Our recent studies have demonstrated that there is a trade-off between active area and peripheral contributions resulting in an optimum PD size, [?]. This fact is essential for small PDs, where the collecting area surrounding the junction becomes comparable to the active area. An analytical model for $\mathrm{p}-\mathrm{n}^{+}$ PD response estimation was developed and validated through device simulations and experimental measurements, [?]. Due to the role of CTK in ever shrinking pixels, its prediction by means of an analytical model would greatly alleviate the pixel design optimization process. Toward this end, Section II presents a CTK analytical model. The model was validated with device simulations with ATLAS and the results are analyzed in Section III. An in-depth analysis by comparison of the model with experimental results in a $180 \mathrm{~nm}$ standard fabrication process can be found in Section IV. Finally, the main conclusions are summarized in Section V. 


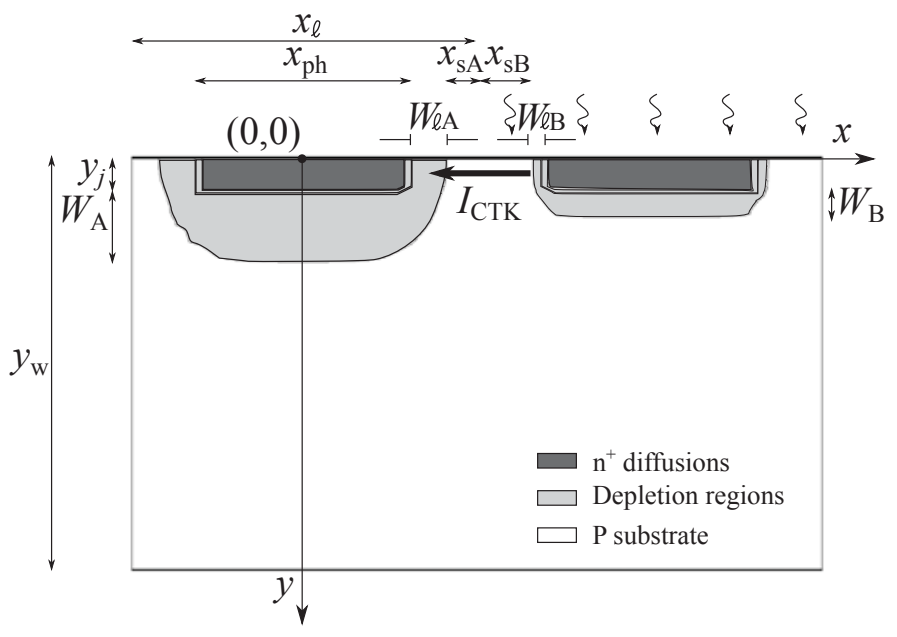

Fig. 1. Cross-section of the three-dimensional photodiode, A (left), and its neighbor, $\mathrm{B}$ (right), along with their geometrical parameters. The $\mathrm{n}^{+}$ diffusions and the photodiodes are $x_{\mathrm{ph}}$ and $x_{\ell}$ wide and $y_{\mathrm{j}}$ and $y_{\mathrm{w}}$ deep, respectively. The depletion regions with thickness $W_{\mathrm{A}, \mathrm{B}}$ (in y-direction) and $W_{\ell_{\mathrm{A}, \mathrm{B}}}$ (in X-direction) are assumed to be located in the substrate because of their lower doping concentration. $x_{\mathrm{SA}} \mathrm{B}$ represent the distance between the edge of the depletion region and the limit of the photodiode for A and B, respectively.

\section{Crosstalk AnAlyticAl MODEL}

Crosstalk (CTK) of a PD is commonly defined as,

$$
\operatorname{CTK}(\%)=\frac{I_{\mathrm{n}}}{I_{\text {ref }}} \times 100
$$

where $I_{\text {ref }}$ is the total photocurrent of the PD of interest under illumination and $I_{\mathrm{n}}$ is the photocurrent measured in the device due to the same illumination exposure of an identical neighbor. Note that both $I_{\text {ref }}$ and $I_{\mathrm{n}}$ are measured in the PD of interest, which operates in the reverse-bias regime. A twodimensional cross-section of a square PD and its neighbor is depicted in Fig. 1, where subscripts A (left) and B (right) represent the $\mathrm{PD}$ of interest, which is reverse-biased, and its zero-biased neighbor under illumination, respectively. The geometrical parameters which describe both structures are also indicated.

The aim of this work is to provide pixel designers with a manageable CTK model for design optimization including the dependencies on the geometrical parameters of the device, the light conditions and process and physical parameters. An analytical model for $I_{\text {ref }}$ has already been proposed and validated through device simulations and experimental results, [?], [?]. Apart from the photocurrent due to the active area illumination, $I_{\text {aa }}$, the model also includes the lateral depletion region and lateral components due to the diffused photocarriers from the surroundings of the junction, $I_{\mathrm{W}}$ and $I_{\text {lateral }}$, [?]. In this work, an analytical expression for $I_{\mathrm{n}}$ is developed following a similar process in a wider structure that takes the neighbor PDs into account, Fig. 1. This current is modeled as the sum of two terms: the diode A reverse-bias saturation current, $I_{0}$, and the CTK lateral current due to carriers generated in the surroundings of a zero-biased neighbor, B, under illumination, $I_{\mathrm{CTK}}$,

$$
I_{\mathrm{n}}=I_{\mathrm{o}}+I_{\mathrm{CTK}}
$$

The minority carrier distribution in the surroundings of the reverse-biased $\mathrm{PD}, \mathrm{A}$, can be found by solving the steady-state two-dimensional continuity equation,

$$
\frac{\partial^{2} N_{\mathrm{A}}}{\partial x^{2}}+\frac{\partial^{2} N_{\mathrm{A}}}{\partial y^{2}}-\frac{N_{\mathrm{A}}}{L_{\mathrm{n}}^{2}}=0
$$

under the following boundary conditions

$$
\begin{aligned}
N_{\mathrm{A}}(x, 0) & =\gamma_{\mathrm{A}} \frac{D_{\mathrm{n}}}{S_{\mathrm{n}}} \\
N_{\mathrm{A}}\left(\frac{x_{\mathrm{ph}}}{2}+W_{\ell_{\mathrm{A}}}, y\right) & =-n_{\mathrm{p} 0} \\
N_{\mathrm{A}}\left(x, y_{\mathrm{w}}\right) & =0 \\
N_{\mathrm{A}}\left(\frac{x_{\ell}}{2}, y\right) & =N_{\mathrm{CTK}}(y)
\end{aligned}
$$

where $N_{\mathrm{A}}, n_{\mathrm{p} 0}, L_{\mathrm{n}}, D_{\mathrm{n}}$ and $S_{\mathrm{n}}$ are the excess electron concentration for device $A$, equilibrium electron concentration, electron diffusion length, electron diffusion coefficient and surface recombination velocity of electrons, respectively. Regarding $\gamma_{\mathrm{A}}$, this is a fitting parameter that was already introduced in our previous model for uniformly illuminated PDs in the visible range, [?]. $N_{\mathrm{CTK}}(y)$ stands for the excess minority carrier distribution in the border between the PDs due to the illumination of its neighbor, $\mathrm{B}$. This function is found by solving the steady-state two-dimensional continuity equation in the surroundings of the neighbor,

$$
\frac{\partial^{2} N_{\mathrm{B}}}{\partial x^{2}}+\frac{\partial^{2} N_{\mathrm{B}}}{\partial y^{2}}-\frac{N_{\mathrm{B}}}{L_{\mathrm{n}}^{2}}=-\frac{G(y)}{D_{\mathrm{n}}}
$$

with appropriate boundary conditions

$$
\begin{aligned}
N_{\mathrm{B}}(x, 0) & =\gamma_{\mathrm{B}} \frac{D_{\mathrm{n}}}{S_{\mathrm{n}}} \\
N_{\mathrm{B}}\left(x_{\ell}-\frac{x_{\mathrm{ph}}}{2}-W_{\ell_{\mathrm{B}}}, y\right) & =0 \\
N_{\mathrm{B}}\left(x, y_{\mathrm{w}}\right) & =0 \\
N_{\mathrm{B}}\left(\frac{x_{\ell}}{2}, y\right) & =N_{\mathrm{CTK}}(y)
\end{aligned}
$$

where $N_{\mathrm{B}}$ is the excess electron concentration for device B, $\gamma_{\mathrm{B}}$ is another fitting parameter and $G(y)=\alpha \Phi_{0} e^{-\alpha y}$ is the optical generation rate, where $\alpha$ is the absorption coefficient and $\Phi_{0}=\frac{P_{\mathrm{opt}} T_{\mathrm{c}} \lambda}{h c}$ is the photon flux penetrating the surface. $P_{\mathrm{opt}}, T_{\mathrm{c}}, \lambda, h$ and $c$ are the incident optical power, transmission coefficient, impinging radiation wavelength, Plank's constant and speed of light, respectively. In addition, the current density in $\mathrm{x}$-direction at the boundary of the depletion region of zerobiased PD B is expected to be negligible,

$J_{\mathrm{nB}}\left(x_{\ell}-\frac{x_{\mathrm{ph}}}{2}-W_{\ell_{\mathrm{B}}}, y\right)=\left.q D_{\mathrm{n}} \frac{\partial N_{\mathrm{B}}(x, y)}{\partial x}\right|_{x_{\ell}-\frac{x_{\mathrm{ph}}}{2}-W_{\ell_{\mathrm{B}}}}=0$

The solution of Equation (3) and Equation (5) under boundary conditions (4) and (6), respectively, is found applying the method of separation of variables,

$$
N_{\mathrm{A}, \mathrm{B}}(x, y)=u_{\mathrm{A}, \mathrm{B}}(x, y)+v_{\mathrm{A}, \mathrm{B}}(y)
$$

Equation (8) is inserted in Equation (3)-(6) giving a secondorder homogeneous, $v_{\mathrm{A}}$, and a non-homogeneous, $v_{\mathrm{B}}$, linear 


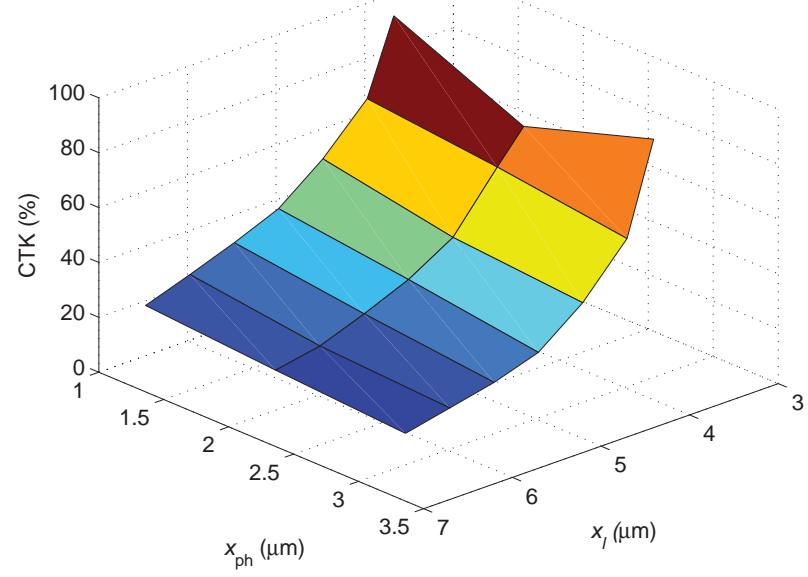

(a) ATLAS device simulations.

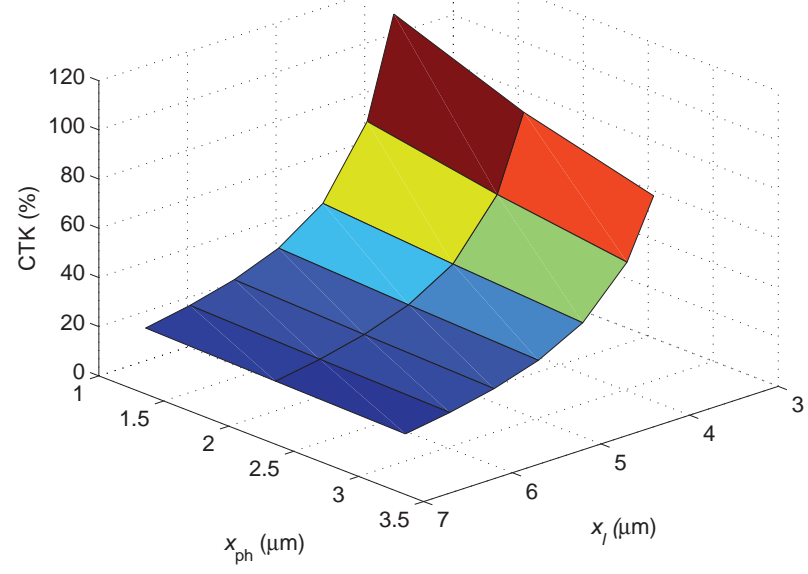

(b) Results given by the model.

Fig. 2. CTK results in terms of geometrical parameters for $\lambda=550 \mathrm{~nm}$.

ordinary differential equations. The former is easily solved,

$$
v_{\mathrm{A}}(y)=\gamma_{\mathrm{A}} \frac{D_{\mathrm{n}}}{S_{\mathrm{n}}} \frac{\sinh \left(\frac{y_{\mathrm{w}}-y}{L_{\mathrm{n}}}\right)}{\sinh \left(\frac{y_{\mathrm{w}}}{L_{\mathrm{n}}}\right)}
$$

whereas $v_{\mathrm{B}}$ is achieved applying the method of variation of constants,

$$
\begin{aligned}
v_{\mathrm{B}}(y) & =\left(\gamma_{\mathrm{B}} \frac{D_{\mathrm{n}}}{S_{\mathrm{n}}}-\frac{\frac{\alpha \Phi_{0}}{D_{\mathrm{n}}} e^{-\alpha y}}{\frac{1}{L_{\mathrm{n}}^{2}}-\alpha^{2}}\right) \frac{\sinh \left(\frac{y_{\mathrm{w}}-y}{L_{\mathrm{n}}}\right)}{\sinh \left(\frac{y_{\mathrm{w}}}{L_{\mathrm{n}}}\right)} \\
& +\frac{\frac{\alpha \Phi_{0}}{D_{\mathrm{n}}} e^{-\alpha y}}{\frac{1}{L_{\mathrm{n}}^{2}}-\alpha^{2}} \cdot \frac{e^{-\alpha y} \sinh \left(\frac{y_{\mathrm{w}}}{L_{\mathrm{n}}}\right)-e^{-\alpha y_{\mathrm{w}}} \sinh \left(\frac{y}{L_{\mathrm{n}}}\right)}{\sinh \left(\frac{y_{\mathrm{w}}}{L_{\mathrm{n}}}\right)}
\end{aligned}
$$

Regarding $u_{\mathrm{A}}$ and $u_{\mathrm{B}}$, they are the solution of a secondorder homogeneous partial differential equation in two independent variables. Assuming a separable solution in the form

$$
u(x, y)=X(x) Y(y)
$$

and applying the boundary conditions at $y=0$ and $y=y_{\mathrm{w}}$,

$$
u_{\mathrm{A}, \mathrm{B}}(x, y)=\sum_{n=1}^{\infty}\left(a_{n_{\mathrm{A}, \mathrm{B}}} e^{\sqrt{\sigma_{n}} x}+b_{n_{\mathrm{A}, \mathrm{B}}} e^{-\sqrt{\sigma_{n}} x}\right) \sin \left(\theta_{n} y\right)
$$

where $\sigma_{n}=\frac{1}{L^{2}}+\theta_{n}^{2}, n=1,2,3, \ldots, \theta_{n}=\frac{n \pi}{y_{\mathrm{w}}}$ and constants $a_{n_{\mathrm{A}, \mathrm{B}}}$ and $b_{n_{\mathrm{A}, \mathrm{B}}}$ are determined from the remaining boundary conditions, which will be satisfied if $v_{\mathrm{A}, \mathrm{B}}(y)$ and $N_{\mathrm{CTK}}(y)$ can be represented by Fourier sine series. In addition, Equation (7) implies that

$$
\left.q D_{\mathrm{n}} \frac{\partial u_{\mathrm{B}}(x, y)}{\partial x}\right|_{x_{\ell}-\frac{x_{\mathrm{ph}}}{2}-W_{\ell_{\mathrm{B}}}}=0
$$

The current density measured in the PD due to the illumination of its neighbor is calculated by deriving the excess electron density in the $x$-direction at the boundary of the depletion region, $\frac{x_{\mathrm{ph}}}{2}+W_{\ell_{\mathrm{A}}}$,

$$
J_{\mathrm{nA}}\left(\frac{x_{\mathrm{ph}}}{2}+W_{\ell_{\mathrm{A}}}, y\right)=\left.q D_{\mathrm{n}} \frac{\partial u_{\mathrm{A}}(x, y)}{\partial x}\right|_{\frac{x_{\mathrm{ph}}}{2}+W_{\ell_{\mathrm{A}}}}
$$

Finally, the lateral current component due to the CTK is found integrating the current density at the boundary of the depletion region over the side-wall,

$$
I_{\mathrm{CTK}}=\int_{-\frac{x_{\mathrm{ph}}}{2}}^{\frac{x_{\mathrm{ph}}}{2}} \int_{0}^{y_{\mathrm{j}}} J_{\mathrm{nA}}\left(\frac{x_{\mathrm{ph}}}{2}+W_{\ell_{\mathrm{A}}}, y\right) \mathrm{d} y \mathrm{~d} z
$$

which leads to the following expression,

$$
\begin{aligned}
& I_{\mathrm{CTK}}=x_{\mathrm{ph}} q D_{\mathrm{n}} \sum_{n=1}^{\infty} \frac{\sqrt{\sigma_{n}}}{\theta_{n}} \\
& \quad \times\left(\frac{V_{\mathrm{B}}\left(\cosh \left(\sqrt{\sigma_{n}} x_{\mathrm{sB}}\right)-1\right)+V_{\mathrm{A}}\left(1-\cosh \left(\sqrt{\sigma_{n}} x_{\mathrm{sA}}\right)\right)}{\sinh \left(\sqrt{\sigma_{n}} x_{\mathrm{sA}}\right)}\right) \\
& \quad \times\left(1-\cos \left(\theta_{n} y_{\mathrm{j}}\right)\right)
\end{aligned}
$$

where

$$
V_{\mathrm{A}}=-\frac{2(-1)^{n} \theta_{n}}{y_{\mathrm{w}}} \frac{\gamma_{\mathrm{A}} D_{\mathrm{n}}}{S_{\mathrm{n}} \sigma_{n}} \cosh \left(\frac{y_{\mathrm{w}}}{L_{\mathrm{n}}}\right)
$$

$$
\begin{aligned}
V_{\mathrm{B}} & =-\frac{2(-1)^{n} \theta_{n}}{y_{\mathrm{w}}}\left(\frac{\gamma_{\mathrm{B}} D_{\mathrm{n}}}{S_{\mathrm{n}} \sigma_{n}} \cosh \left(\frac{y_{\mathrm{w}}}{L_{\mathrm{n}}}\right)\right. \\
& \left.+\frac{\frac{\Phi_{0} \alpha}{D_{\mathrm{n}}}}{\frac{1}{L_{\mathrm{n}}^{2}}-\alpha^{2}}\left(\frac{\sinh \left(\alpha y_{\mathrm{w}}\right)}{\alpha^{2}+\theta_{n}^{2}}-\frac{\cosh \left(\frac{y_{\mathrm{w}}}{L_{\mathrm{n}}}\right)-e^{-\alpha y_{\mathrm{w}}}}{\sigma_{n}}\right)\right)
\end{aligned}
$$

and $x_{\mathrm{sA}, \mathrm{B}}=\frac{x_{\ell}}{2}-\frac{x_{\mathrm{ph}}}{2}-W_{\ell_{\mathrm{A}, \mathrm{B}}}$. The summation in Equation (16) is simplified because $n=1$ proved to be appropriate.

Making use of the modeled CTK current component in addition with the PD model proposed in previous works, [?], the final CTK model is given by

$$
\operatorname{CTK}(\%)=\frac{I_{\mathrm{o}}+I_{\mathrm{CTK}}}{I_{\mathrm{aa}}+I_{\mathrm{W}}+I_{\text {lateral }}} \times 100
$$



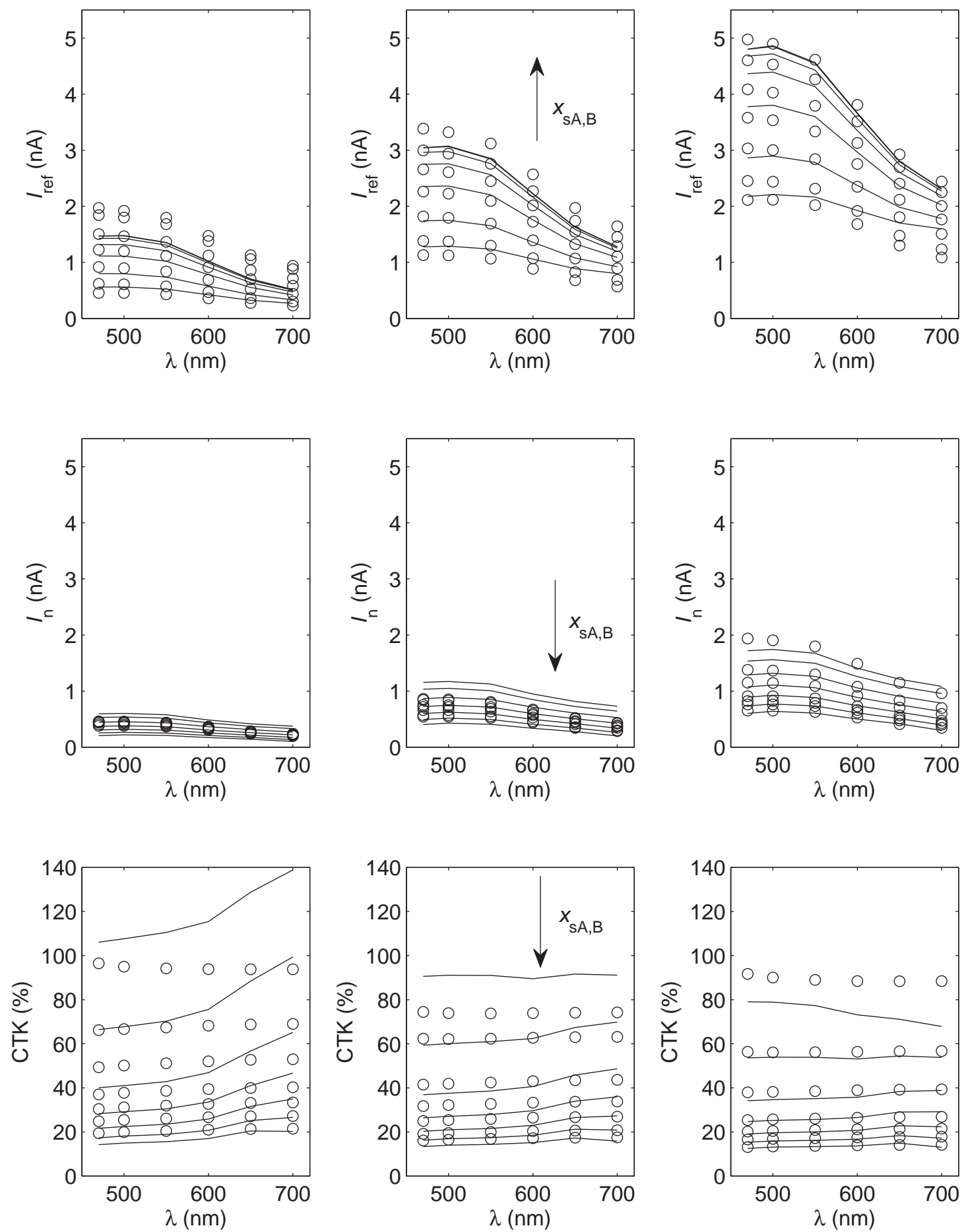

Fig. 3. Spectral results given by the model (lines) and device simulations (circles) for $\mathrm{p}-\mathrm{n}^{+}$junctions with $x_{\mathrm{ph}}=1.06 \mu \mathrm{m}$ (left), $x_{\mathrm{ph}}=2.06 \mu \mathrm{m}$ (middle), $x_{\mathrm{ph}}=3.06 \mu \mathrm{m}$ (right) and $x_{\mathrm{SA}, \mathrm{B}}+W_{\ell_{\mathrm{AB}}}=0.35,0.50,0.75,1.00,1.25,1.50,1.75,2.475 \mu \mathrm{m}$. $I_{\text {ref }}$ is the total photocurrent of the PD of interest (A) under illumination and $I_{\mathrm{n}}$ is the photocurrent measured in the device due to the same illumination exposure of an identical neighbor (B). $x_{\mathrm{SA}, \mathrm{B}}$ represent the distance between the edge of the depletion region and the limit of the photodiode for $\mathrm{A}$ and $\mathrm{B}$, respectively. 


\section{DEVice Simulations}

Three-dimensional device simulations using ATLAS from Silvaco were performed in order to validate the developed model. A pair of identical $\mathrm{p}-\mathrm{n}^{+}$junction PDs was simulated under uniform illumination in the visible range impinging perpendicularly onto the top surface. Thus, $I_{\text {ref }}$ and $I_{\mathrm{n}}$ are obtained by illumination of reverse-biased device $\mathrm{A}\left(-\frac{x_{\ell}}{2} \leq x, z \leq \frac{x_{\ell}}{2}\right)$ and zero-biased PD B $\left(\frac{x_{\ell}}{2} \leq x \leq \frac{3 x_{\ell}}{2},-\frac{x_{\ell}}{2} \leq z \leq \frac{x_{\ell}}{2}\right)$, respectively.

The fitting parameters $\gamma_{\mathrm{A}}$ and $\gamma_{\mathrm{B}}$ of the boundary conditions in Equations (4) and (6) were found by comparison with simulation data resulting in functions of both the incident optical power, $P_{\mathrm{opt}}$, and the radiation wavelength,

$$
\gamma_{\mathrm{A}, \mathrm{B}}=P_{\mathrm{opt}} \sum_{i=0}^{4} C_{\mathrm{A}_{i}, \mathrm{~B}_{i}} \lambda^{i}
$$

where $C_{\mathrm{A}_{i}, \mathrm{~B}_{i}}$ are fitting constants.

Fig. 2 depicts the simulated (a) and modeled (b) CTK for $\lambda=550 \mathrm{~nm}$ and different values of the PD diffusion size, $x_{\mathrm{ph}}$, and total device width, $x_{\ell}$, including the smallest PD allowed by the design rules of a $180 \mathrm{~nm}$ CMOS standard technology. These surface plots show a good agreement, revealing the CTK dependence on both the diffusion and total PD area, increasing as both parameters decrease. This conclusion is in accordance with previous results in the literature, [?]. It is interesting to highlight that the influence of the distance between the edge of the depletion region and the limit of the photodiode, $x_{\mathrm{SA}, \mathrm{B}}$, proves to be more important than the dependence on the diffusion size, $x_{\mathrm{ph}}$. In other words, CTK increases dramatically as the surface area surrounding the junction decreases.

One of the most interesting parameters to take into consideration in the model development process is the wavelength of the light source, as image sensors operate within a specific illumination range. According to the proposed model, the spectral response within the visible range was also studied, see Fig. 3. In this figure, $I_{\text {ref }}, I_{\mathrm{n}}$ and CTK curves obtained with the model and the simulator are represented in lines and circles, respectively. PDs with a diffusion width, $x_{\mathrm{ph}}$, of $1.06 \mu \mathrm{m}$ (left column), $2.06 \mu \mathrm{m}$ (middle column) and $3.06 \mu \mathrm{m}$ (right column) are compared. For each PD, different values of the distance between the edge of the depletion region and the limit of the photodiode, $x_{\mathrm{SA}, \mathrm{B}}$, were considered. Note that the arrows indicate the increasing direction of $x_{\mathrm{SA}, \mathrm{B}}$, which is not the same for $I_{\text {ref }}, I_{\mathrm{n}}$ and CTK plots. The modeled CTK fits the simulation data and presents a minor disagreement for PDs with the smallest values of the the distance between the edge of the depletion region and the limit of the photodiode, $x_{\mathrm{sA}, \mathrm{B}}$, under long wavelengths. This corresponds to the slight difference already observed in the $I_{\text {ref }}$ model, [?]. As can be seen in Fig. 2, these are the devices which present the highest level of CTK. In fact, inspection of Fig. 3 demonstrates that $I_{\mathrm{n}}$ tends to $I_{\mathrm{ref}}$ as $x_{\mathrm{SA}, \mathrm{B}}$ shrinks, which means that CTK increases whereas the distance between diffusions decreases. Finally, neither $\lambda$ nor $x_{\mathrm{ph}}$ present a significant effect on CTK for the considered structures.

This kind of PD characterization is essential from the point of view of the device designer. The accurate knowledge of the

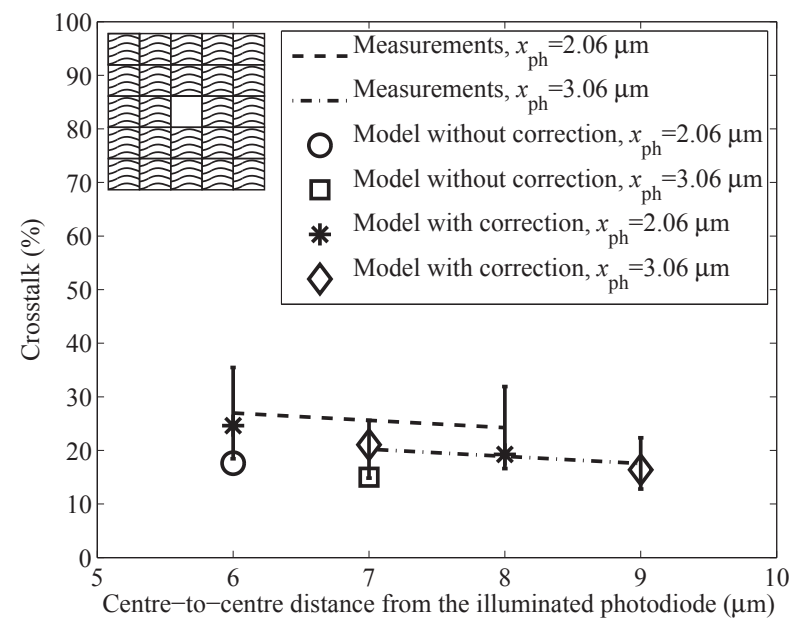

Fig. 4. Scheme of the $5 \times 5$ test arrays of photodiodes for CTK measurements (upper left corner inset). The lines represent the CTK experimental measurements of two PDs with different diffusion width, $x_{\mathrm{ph}}$, due to the illumination of the neighbors in the cardinal and diagonal directions $\left(x_{\mathrm{sA}, \mathrm{B}}=1.75 \mu \mathrm{m}\right.$, $\lambda=660 \mathrm{~nm})$. The symbols represent the CTK given by the developed model, including the correction and extension for the PD in the diagonal.

CTK dependencies on the geometrical features of the structure can be used to maximize the PD response whereas keeping an acceptable level of CTK, [?]. It is important to note that this is an approximation to the maximum expected CTK, that is, the worst case scenario consisting of two PDs without extra neighbors. However, in a real array implementation the rest of neighbors in the vicinity and the in-pixel electronics are expected to reduce CTK slightly.

Finally, some remarks are needed for the sake of clarity. Regarding the considered structure, square-shaped PDs were used for simplicity but without loss of generality. In addition, the model was developed for $\mathrm{p}-\mathrm{n}^{+}$junction PDs, but it could be easily extend to consider other junctions.

\section{EXPERIMENTAL MEASUREMENTS AND DISCUSSION}

Further analysis of the model accuracy was performed by comparison with experimental measurements. Test structures in a standard $180 \mathrm{~nm}$ fabrication process were characterized under uniform illumination in the visible range impinging perpendicularly onto the top surface. They consist of $5 \times 5$ arrays of identical $\mathrm{p}-\mathrm{n}^{+}$PDs with a metal stack blocking the penetration of light except for the central device, as can be seen in the scheme in the upper left corner inset of Fig. 4. Then, the electrical CTK in the neighboring PDs due to the illumination of the one at the center of the array was observed. Only one of the adjacent PDs and other in the diagonal were measured, whereas the second ring was placed in order to guarantee that the characterized PDs were surrounded by identical devices. To measure each device independently, each PD is directly connected to a raw pad without ESD protection in order to avoid unwanted capacitance coupling with the sensing node.

The experimental set-up consists of a laser, a mirror, a microscope objective lens, a platform, a Printed Circuit Board (PCB) and a picoammeter/voltage source. The incoming laser 
beam is redirected towards the surface of the Device Under Test (DUT) by a mirror oriented at $45^{\circ}$ to the beam. The DUT consists of a packaged sample of the fabricated chips, which fits a plastic socket in the PCB. In order to center the PD of interest under the laser beam, the board is fixed to a two-axis platform. After positioning, the PD is reverse-biased and the current is read by a picoammeter/voltage source.

Two PDs with different diffusion width, $x_{\mathrm{ph}}$, and $x_{\mathrm{sA}, \mathrm{B}}=$ $1.75 \mu \mathrm{m}$ were characterized under a light source with $\lambda=$ $660 \mathrm{~nm}$. A set of 19 chips was considered for the statistics. The CTK results of two neighbors in the cardinal and diagonal directions are depicted in Fig. 4 connected with lines and with their corresponding errorbar. The data suggest that CTK is expected to decay with distance from the illuminated PD, whereas the diffusion width, $x_{\mathrm{ph}}$, seems to have less impact on this phenomenon. The CTK decay with distance was previously reported in the literature, [?], whereas both the proposed model and device simulations described in previous sections have also revealed a weak CTK dependence on $x_{\mathrm{ph}}$.

Along with the experimental data, two points marked with a circle and a square in Fig. 4 represent the CTK given by the developed model for the same structures. CTK of the PDs in the diagonal direction are not plotted because the model only considers two adjacent PDs. It is observed that the model underestimates CTK compared with the experimental results, but the model results are almost within the error interval. In this sense, it is important to note that the measured arrays of PDs include a metal stack blocking the penetration of light except for the central device. This set-up is necessary in order to evaluate CTK, but it will obviously not be present in a real array. The main problem of this technique is that the hole in the center of the array may cause diffraction and thus an increase in CTK. This is an optical CTK component which will not occur in a real situation and therefore it is not considered by the proposed model, but it must be taken into account in order to compare the modeled CTK with the experimental results. Therefore, although the model only accounts for the diffused photocarriers collected through the side-wall of the depletion region which is opposite the illuminated PD, collection through the other side-walls due to diffraction effects is also expected in the test structures as they are placed in a $5 \times 5$ array. This fact can be easily introduced in the model modifying the limits of integration over $z$ in

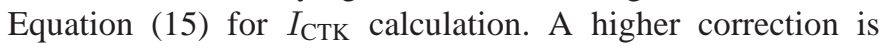
expected for the PD in the diagonal direction because two sidewalls of the depletion region are potential carrier collectors. The results given by the model using $1.4 x_{\mathrm{ph}}$ and $1.9 x_{\mathrm{ph}}$ in Equation (15) for PDs in the cardinal and diagonal directions, respectively, are given in Fig. 4 . The extension of the model for the PD in the diagonal was done taking $\sqrt{2} x_{\mathrm{SA}, \mathrm{B}}$ as the half distance between the diffusion corners. As can be seen, the model proves to be a comprehensive tool for CTK prediction in $\mathrm{p}-\mathrm{n}^{+}$CMOS PDs.

\section{CONCLUSIONS}

An analytical model for CTK estimation in $\mathrm{p}-\mathrm{n}^{+}$CMOS PDs under normal incidence uniform illumination in the visible range has been reported. Despite the mathematics involved in the problem, a compact, closed-form, manageable expression in terms of light conditions and physical, geometrical and process parameters is achieved, which is of great interest to engineers for pixel design optimization and circuit simulation. An extensive validation of the model has been performed by comparing with simulation results and experimental data.

\section{ACKNOWLEDGMENT}

The authors would like to thank the collaboration of professor Dr. Vicente Moreno de las Cuevas from the Department of Applied Physics of the University of Santiago de Compostela. 
Beatriz Blanco-Filgueira received the degree in physics and the Ph.D. degree from the University of Santiago de Compostela, Spain, in 2007 and 2012, respectively.

She is currently a postdoctoral researcher at CiTIUS and her research interests are the modeling of integrated circuits and semiconductor devices.

Paula López Martínez received the degree in physics and the Ph.D. degree from the University of Santiago de Compostela, Spain, in 1998 and 2003, respectively.

She is currently an Associate Professor at the University of Santiago de Compostela and author or coauthor of more than 50 research papers. Her current research interests include design of mixed signal integrated circuits and physical modeling of electronic devices.

Juan Bautista Roldán Aranda received the degree in physics and the Ph.D degree from the University of Granada, Spain, in 1993 and 1997, respectively.

He is currently an Associate Professor at the University of Granada. His current research interests include modeling of nanometric conventional and multigate devices and Verilog-A implementation of device models for circuit simulation. He is the author or coauthor of more than 60 refereed technical journal papers and 70 communications at international conferences. 